\title{
DIS: An n-channel tachistoscope algorithm
}

\author{
D. J. K. MEWHORT \\ Queen's University at Kingston, Kingston, Ontario K7L 3N6, Canada
}

DIS is a stand-alone, file-oriented, $n$-channel tachistoscope simulator. The paper describes the program in terms of both the syntax required to set up a display and the basic operation of the algorithm. Short routines illustrating two experimental paradigms are provided.

DIS is a stand-alone, file-oriented, n-channel tachistoscope simulator. Optionally, it can read response buttons, calculate reaction time latencies, transcribe alphanumeric responses, and create a field recording the data. The algorithm is coded in FORTRAN, but assembly language routines handle real-world interfaces. It has been implemented on a PDP-8/e running under OS/8 and on a PDP-11 running under RT-11.

\section{THE CHARACTER SET}

The algorithm displays characters defined in dotmatrix form. The characters must be predefined and associated with a character name. Each name must be a single ASCII character.

A separate routine, CARGEN (character generator), is used to prepare a character set. CARGEN accepts a dot pattern and creates an indexed file listing the character names and the dot-address values associated with each.

There are no formal restrictions concerning the dotmatrix size. Further, any dot pattern (e.g., alphanumerics, faces, or geometric forms) can be used. When using characters defined in very large matrices, however, memory restrictions may limit the number of different characters available simultaneously. The illustrations that follow assume an alphanumeric character set, but another set could serve as well.

\section{INPUT SYNTAX}

On each trial, DIS reads a description of the material to be shown; Table 1 illustrates the syntax. Each trial consists of one or more character strings. A character string represents one field of an optical tachistoscope;

The work described here was supported by a grant from the National Research Council (AP-318) and by grants from the Advisory Research Council, School of Graduate Studies and Research, Queen's University. It is a pleasure to acknowledge the assistance of both agencies. It is also a pleasure to acknowledge the assistance of A. J. Campbell, Rainer von Königslöw, and M. Withey during many discussions of display and programming technique. Finally, I thank Gordon Logan for help adapting the original PDP-8 algorithm to the PDP-11. Address correspondence to D. J. K. Mewhort, Department of Psychology, Queen's University, Ontario K7L 3N6, Canada. that is, it lists characters appearing simultaneously on the display. Each string is read from one record of a predefined file.

Two special characters, the period and the comma, are used as string delimiters. The period (.) marks the end of the final string of the trial, and the comma (,) ends all other strings in the trial.

The record following each character string indicates the string duration and, when relevant, the interstimulus interval to the subsequent string. The latter values are expressed as decimal integers in two five-column fields; the temporal units are arbitrary and default to milliseconds.

Two formatting conventions determine the location of each character on the display. The first, a withinstring convention, determines the position of each character relative to the others in the string. The second concerns the position of the whole string relative to the center of the monitor. The latter specifications are external to the input syntax and are supplied during the user dialogue, a feature described later.

To implement within-string formatting, DIS assumes that characters occupy separate positions on one or more rows. Three formatting features are used to locate each character relative to the leftmost character in the top row of the string. First, a slash $(/)$ indicates a leftjustified skip to a new row on the display. Second, an

Table 1

An Illustration of DIS Input Syntax

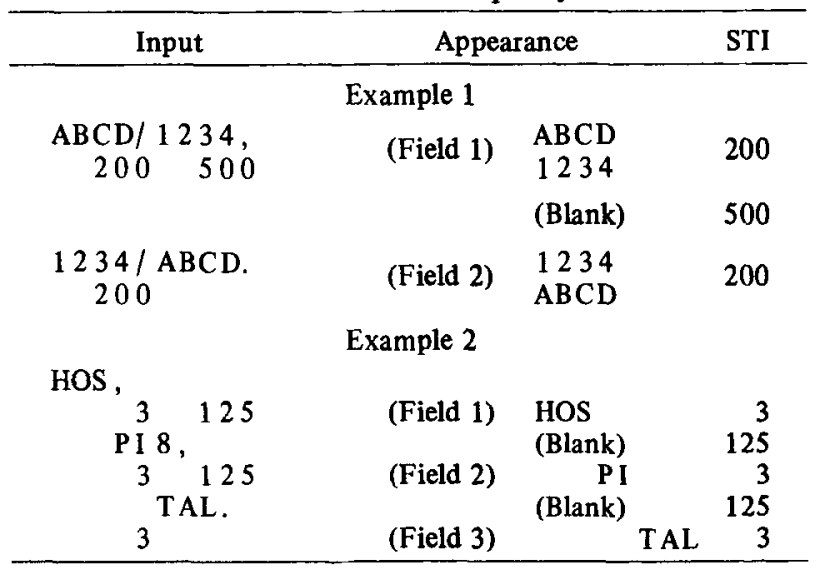

Note-STI = successive time intervals in milliseconds. 
"illegal" character name, that is, one not included in the current character set, is treated as a blank. In such cases, DIS adjusts the position of the next "legal" character by shifting it one character width to the right. Third, the user can declare a "legal" character to be null. When a null character is used, it shifts the position of other characters in the same way as a blank. The null character, however, also changes the brightness of the string, a point to be elaborated later.

\section{PROGRAM OPERATION}

DIS assumes that an experiment consists of a series of trials. At the start of an experiment, DIS interacts with the user to acquire a number of parameters. Once established, the parameters cannot be changed without restarting the program. The latter restriction is not a serious one; modest changes to the coding would permit most parameters to be modified during an experiment.

\section{User Dialogue}

The exact form of the dialogue varies. Experience in our laboratory suggests that an exhaustive dialogue is too time consuming. Instead, we default a number of parameters and have configured appropriate versions of the basic routine to suit particular experimental paradigms. For example, DISRT is a version of DIS configured to "listen" for a response button following the final string of each trial and to create a file recording both the particular response and its latency. DISNLR expects to read alphanumeric responses from a remote keyboard following the final string of a trial and records the responses in a file. Thus, DISRT is used for manual response, forced-choice tasks, and DISNLR is used for verbal response, free recall tasks.

Even with a number of default parameters, some material must be read interactively. The latter includes a file name for stimuli, a file name for responses, the number of trials in the experiment, a null character, and some display-format information. Table 2 lists the main parameters organized in terms of typical defaults.

The formatting parameters deserve a special note. Recall that the input syntax determines the position of characters relative to each other within the character string. At the start of an experiment, however, the user must specify the position of the whole string relative to the physical center of the monitor. To do so, the user specifies the location of the top- and leftmost character relative to the center of the monitor. Specification is in terms of row and character-position units. Thus, to obtain a centered display for the first example in Table 1, one would request two characters to the left of center and one row above center.

Specification in terms of character and row units may seem unusual. To understand such units, however, a novice need not understand any details of the digitalto-analog (D/A) control unit. As a result, novice users
Table 2

DIS Parameters Illustrating Typical Default Conventions

Version Defaults

DISRT -a version that expects to read response buttons at the end of the last string of each trial. It also computes response latencies and creates a file recording the response and the latency.

DISNLR - a version that expects to read alphanumeric responses from a remote keyboard after the last string of each trial. It also creates a file recording the responses.

\section{Internal Defaults}

CHAR43 -a file name for the current character set.

NCHAR - the number of characters in the current character set.

NROW -the number of rows in the dot matrix defining each character.

NCOLS - the number of columns in the dot matrix.

NSPACE -the spacing between characters, expressed in terms of the number of columns in the dot matrix defining each character.

INSCALE - the number of bits between adjacent dots when an all-dot character is used.

IROWDR - the spacing between rows, expressed in terms of the number of columns in the basic dot matrix.

ENABLE -a machine-specific value determining the clock rate.

\section{Active Parameters}

FILE -a file name for the display data.

FILEO -a file name for response data.

NUNIT -logical (FORTRAN) unit number specifying a physical device for the stimulus file.

NDOT - the number of character positions to the left of the physical center of the display monitor.

YY - the number of character rows above the physical center of the display monitor.

ITR -a switch selecting default values or an extended user dialogue.

NT -the number of trials for the experiment.

(e.g., undergraduates) find the convention easy to use.

Typically, the fixation dot is located at the center of the monitor. Its position is flexible, however, and one must either accept the default or indicate its position (in bits) relative to the center of the monitor.

\section{Display Procedure}

On each trial, DIS reads all of the information describing each string of the trial. The material is interpreted in terms of the syntax described earlier, and an address vector is calculated that stores real-display dot coordinates for each dot of the trial. When calculating the address vector, each string is treated independently, and successive strings follow each other in the vector. In addition, pointers are calculated that index the start position for successive strings in the address vector. Finally, the string durations and the interstring intervals are stored in separate vectors.

For each string, the first entry in the address vector 
is the ordinate value for the topmost row of the string. The next entries are abscissa values for all dots with that ordinate reading from left to right. The next entry is a row delimiter. The latter is followed by the ordinate for the next row of dots, and it is followed, in turn, by the abscissa values for all dots on that row, reading from right to left. The address vector continues until all dots in the trial are represented.

After the address vector has been calculated, DIS brightens a fixation point and waits for the subject to initiate the trial by pushing a start button. Once the start button has been "heard," a clock used to time the display is started, and control is passed to a short assembly language dot-brightening subroutine.

The dot-brightening routine uses the appropriate pointer to find the start position in the address vector and proceeds through the vector brightening the dots. Because of the organization of the address vector, the dots in each string are brightened in a row-by-row order starting from the top of the string. Further, dots within successive rows are brightened in an alternating left-to-right, right-to-left order. The display subroutine refreshes the whole string continuously until interrupted by the clock.

The top-to-bottom alternating-direction display order is used to minimize fly-back dot-displacement problems. When a D/A point-plot apparatus is driven at very high speed, large dot displacements are associated with inaccurate dot positioning on the screen. To correct the situation, one must either slow the rate of presentation of successive dots or minimize the successive dot displacements.

Because each string is refreshed continuously throughout the display, a small display, one involving a small number of points, will be refreshed more frequently than a large one. Consequently, a small display will appear brighter than a large one. To maintain equal brightness across strings of different lengths, DIS uses a null character. When the null character is encountered, DIS inserts the appropriate values in the address vector, but none of the dots associated with the null are brightened. The time required to process the null character during the display, however, is identical to that for the character when it is used normally. Thus, by inserting null characters appropriately, the experimenter can maintain constant brightness across strings of different lengths.

The second example in Table 1 treats the 8 as a null character. Without the null character, the two-letter syllable would be slightly brighter than the three-letter syllables involved in the trial.

The null character serves another purpose. When one trial has been displayed, DIS reads and prepares the data for the next trial. When ready for the trial, DIS signals its preparedness by re-presenting the fixation dot. Using an RK05 disk, the preparation is fast; a 16 character ( 5 by 7 matrix) display takes about $1 \mathrm{sec}$.
In some cases, the fixation point returns too quickly; that is, it acts as a distraction. For such cases, a string of null characters can be appended to the trial. When a string of null characters is appended to the trial, the timing associated with the null string can be used to control the intertrial interval.

The procedure used to distinguish dots associated with the null character may be of some interest. A typical point-plot control uses fewer bits than are available in a full memory word. As a consequence, each value calculated for the address vector has, in effect, two sign bits, the arithmetic bit used by the CPU, and the leftmost bit used by the point-plot control. Because the point-plot control does not use it, the arithmetic sign bit is available as a simple switch. DIS uses the switch to identify a dot associated with the null character.

Several features of the procedure deserve emphasis. First, the routine minimizes CPU decisions during the display. Second, because of the alternating left-to-right, right-to-left dot-display order, the routine minimizes fly-back dot-displacement problems. Both features concern the speed of the display; DIS permits material to be shown as rapidly as possible. Because the routine is fast, it minimizes flicker for large displays, even when using a rapid-decay phosphor. Such phosphor is needed, of course, when accurate control over display duration is required. Finally, the row-by-row display order within a string eliminates the sequential artifacts associated with character-by-character systems. The latter point is particularly important for experiments concerning reading; reading mechanisms appear to be disturbed with character-by-character displays, even at fairly fast presentation rates (e.g., Mewhort, 1974; Mewhort \& Beal, 1977).

\section{Response Recording}

As mentioned earlier, different versions of the basic algorithm have been configured to record various kinds of responses. DISRT records and times manual buttonpress decisions, and DISNLR records ASCII characters read from a remote keyboard. In both cases, the responses are stored in an output file together with various parameters identifying the trial.

At present, DIS does not allow an interactive response-contingent display. Modest changes to the coding, changes exploiting random-access files, would permit a response-contingent feature to be added. Similarly, DIS does not, at present, offer subjects feedback during an experiment. Again, modest changes to the coding would permit the feature to be added.

\section{IMPLEMENTATION NOTES}

A file-oriented system includes some advantages and some liabilities. A short program is usually required to generate a stimulus file. One major advantage of the 


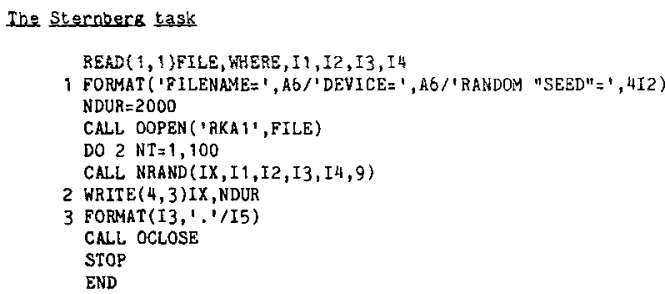

LOOP FOR 3 GROUPS DO 1000 NGRP $=1,3$

CEAD GROUP DURATION \& 3 INTER-STIMULUS INTERVALS READ (1,50) IDUR, ISI

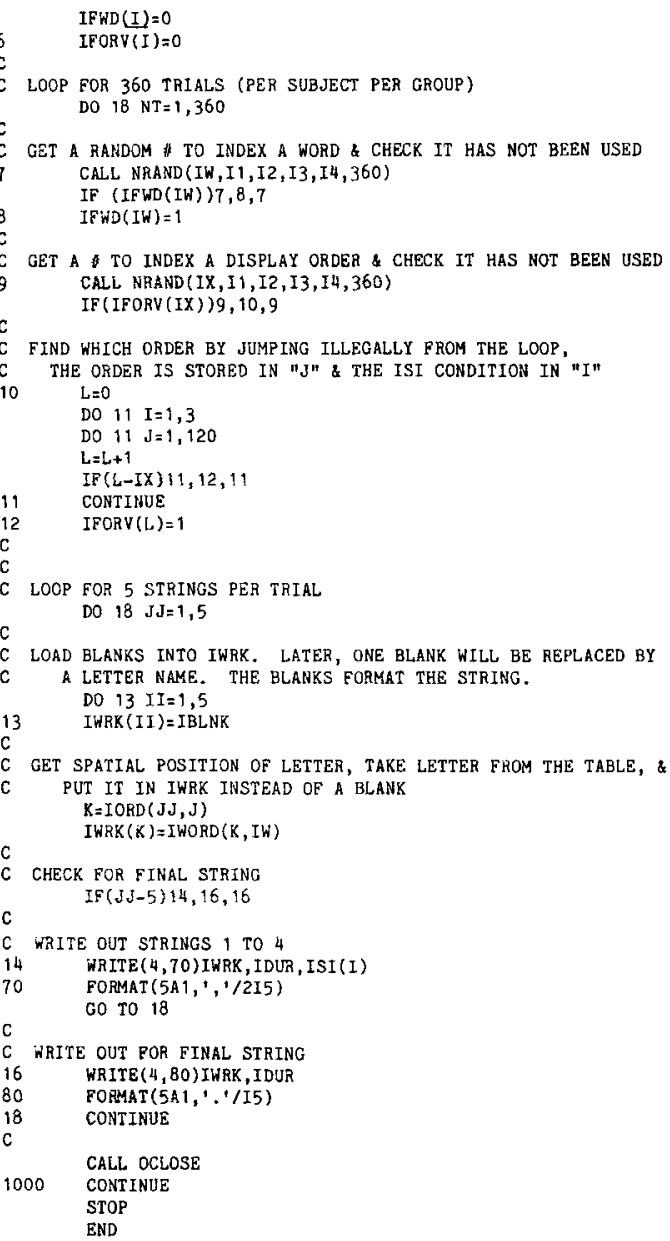

file-oriented system concerns the bugs found in almost all attempts at programming. When in doubt about a particular trial, one can always print the file describing the trial and, thereby, examine the exact nature of the display. Some of the liabilities include the relative inflexibility associated with a predetermined experiment. From a very practical point of view, the major disadvantage concerns the relatively high cost associated with local mass-storage devices. Our solution to the latter problem may be of some interest.

At Queen's University, an interactive terminal system provides convenient access to the main university computer. The system supports asynchronous data lines running at respectable speeds (e.g., 4,800 baud). Accordingly, we have written software to connect the laboratory machine to the main utility. The trick is to make the laboratory machine look like a terminal to the central machine, and to make the latter look like a peripheral to the laboratory machine.

The ability to transfer files back and forth (at reasonable speeds and minimal costs) has proven to be one of the most important features of the laboratory.
In addition to the obvious features (high-speed line printers, tape, and disk facilities), large machines support a variety of software: Typical small-machine operating systems simply do not support the kind of errordiagnosis capability associated with larger systems. Similarly, small systems do not support the range of languages common to large systems. As a consequence, it is usually easier to prepare stimulus files on the large machine and to transfer the file to the small machine. For the same reasons, data analysis is much easier on a large machine.

\section{Stimulus Preparation}

While it is usually easier to prepare a DIS stimulus file on a larger machine, two examples for a PDP-8 are presented in Figure 1. The examples are coded in FORTRAN II and assume the OS/8 operating system. The first example supplies files for a Sternberg (1966) task; the second generates files for an experiment on "perceptual blanking" (Mewhort, Hearty, \& Powell, 1978).

Both examples use a randon-number subroutine, 
NRAND. The subroutine retums a random digit, IX, in response to an eight-digit seed (I1, I2, I3, I4). The final argument specifies the maximum digit to be returned. In addition, the second example assumes two files. The first, ORDERS, is a table of all 5! permutations of the first five integers. The second is a table of 360 fiveletter pseudowords.

\section{REFERENCES}

MewhorT, D. J. K. Accuracy and order of report in tachistoscopic identification. Canadian Journal of Psychology, 1974, 28, 383-398.

MewhoRT, D. J. K., \& BEAL, A. L. Mechanisms of word identification. Journal of Experimental Psychology: Human Perception and Performance, 1977, 3, 629-640.

Mewhort, D. J. K., Hearty, P. J., \& Powell, J. E. A note on sequential blanking. Perception \& Psychophysics, 1978 , 23, 132-136.

StERNBERG, S. High-speed scanning in human memory. Science, 1966, 153, 652-654.

\section{NOTE}

1. For the PDP-8, a program written in "8K FORTRAN" (a version of FORTRAN II) can include both assembly language (SABR) statements and machine-code (octal) instructions. The combination of an upper level language along with the real-time features of the assembler provides a very powerful tool. Within the same program module, one can deal with standard but tedious chores with FORTRAN (e.g., reading from a disk) but handle real-time tasks with the assembly language. Unfortunately, an equivalent feature does not seem to be available for the PDP-11. The PDP-8 version of the program is in the FORTRANSABR combination. The PDP-11 version involves separate MACRO/11 routines that must be assembled independently and linked externally to the FORTRAN module. 